\title{
Photodynamic Action in Thin Sensitized Layers: Estimating the Utilization of Light Energy
}

\author{
Gennady Meerovich ${ }^{1 *}$, Igor Romanishkin ${ }^{1}$, Ekaterina Akhlyustina ${ }^{2}$, Marina Strakhovskaya ${ }^{3,4}$, \\ Evgeniya Kogan ${ }^{5}$, Ivan Angelov ${ }^{6,7}$, Victor Loschenov ${ }^{1,2}$, and Ekaterina Borisova ${ }^{7}$ \\ ${ }_{1}^{1}$ Prokhorov General Physics Institute of the Russian Academy of Sciences, 38 Vavilova str., Moscow 119991, Russia \\ ${ }^{2}$ National Research Nuclear University 'MEPHI', 31 Kashirskoe shosse, Moscow 115409, Russia \\ ${ }^{3}$ Department of Biology, Lomonosov Moscow State University, 1-12 Leninskie Gory, Moscow, 119234, Russia \\ ${ }^{4}$ Federal Research and Clinical Center of Specialized Medical Care and Medical Technologies of the Federal Medical \\ and Biological Agency of Russia, 28 Orekhovyy Bul'var, Moscow 115682, Russia \\ ${ }^{5}$ I. M. Sechenov First Moscow State Medical University, 2-4 Bolshaya Pirogovskaya str., Moscow 119991, Russia \\ ${ }^{6}$ Institute of Organic Chemistry with Centre of Phytochemistry, Bulgarian Academy of Sciences, 9 acad. Georgi \\ Bonchev str., Sofia 1113, Bulgaria \\ ${ }^{7}$ Institute of Electronics, Bulgarian Academy of Sciences, 72 Boulevard Tzarigradsko shose, Sofia 1784, Bulgaria \\ *e-mail: meerovich@mail.ru
}

\begin{abstract}
The result of photodynamic action significantly depends on the density of the light dose absorbed by the photosensitizer. The efficiency of using light to excite photosensitizer molecules and minimization of its loss plays an important role in ensuring the overall success of the process. When carrying out photodynamic treatment of thin sensitized layers (such as inactivation of surface pathogens or in vitro screening studies of photosensitizers), only a part of the light dose is absorbed in the layer, while a significant part is lost, especially at low concentrations of the photosensitizer. In this work, we evaluate the decrease in absorbed light dose depending on the extinction and concentration of the photosensitizer in a thin sensitized layer, the shape of its absorption spectrum, and the shape of the excitation light source spectrum. It was found out that a significant loss of the absorbed dose occurs upon excitation of photosensitizers, especially with low extinction, when using light sources with a broad emission spectrum. This loss must be taken into consideration when predicting the results of photodynamic exposure and optimizing its tactics. (C) 2021 Journal of Biomedical Photonics \& Engineering.
\end{abstract}

Keywords: photodynamic action; relative absorbed photodynamic dose; laser diode; photosensitizer.

Paper \#3436 received 24 May 2021; revised manuscript received 11 Jun 2021; accepted for publication 11 Jun 2021; published online 28 Jul 2021. doi: 10.18287/JBPE21.07.040301 [Special issue on Ekaterina Borisova's contribution to World Biophotonics].

\section{Introduction}

Photodynamic action (PDA) is widely used for the therapy of neoplasms [1,2], infected wounds and other pathological foci of various etiologies [3-5], disinfection of biological fluids, blood components, and water [6]. The photodynamic effect is the photooxidative destruction of molecular structures that ensure the functioning of tumor cells and pathogens (bacteria, viruses) by reactive oxygen species (ROS). Molecules of a photosensitizer (PS) that sensitize the environment in the affected area catalyze the generation of ROS in their vicinity upon excitation with light. The light transfers the energy necessary for this process to the medium containing the photosensitizer. The effect of PDA increases with the volume density of the energy absorbed by PS, which depends on the light intensity, the concentration and extinction of PS. 
Light-emitting diodes (LED) and lasers, primarily on laser diodes (LD), are widely used as light sources for PDA. These light sources provide radiation with high intensity, and their emission spectrum is narrower $\left(\Delta \lambda_{\text {LED }}=20-30 \mathrm{~nm}, \Delta \lambda_{\mathrm{LD}}=4 \mathrm{~nm}\right)$ than the absorption bands of PS. Such light sources can be created, in general, with practically any wavelength (at least in the red and near-infrared spectral ranges) suitable for the excitation of PS. Broadband light sources with a spectral band selected by a bandpass filter in the PS absorption region can also be used for the excitation of PSs [7]. However, only the part of the light energy that lies in the spectral region of the PS absorption band is absorbed. The rest of the light passes through the sensitized medium.

Many PDA problems require the PS excitation in thin $(\leq 5 \mathrm{~mm})$ layers of sensitized aqueous compositions. Such problems include photodynamic inactivation of pathogens - viruses or bacteria, on various surfaces $[8,9]$, in vitro screening studies of PS properties $[10,11]$. For small layer thicknesses and low (less than $10 \mu \mathrm{M}$ ) PS concentrations used for such a PDA, a significant part of the light in the spectral range of the PS absorption band can pass through the layer without participating in the PDA process. This loss, which can be described as a decrease of relative absorbed photodynamic dose (RAPD) [12], results in decreased PDA efficiency. It can also lead to errors in screening studies of PSs with various extinction and concentration since both the losses and the amount of the absorbed energy in the sensitized medium for different PSs will be different.

\section{Materials and Methods}

In this work, such an assessment was carried out for aqueous solutions of cationic PS, which are distinguished by high values of extinction and quantum yield of ROS generation: methylene blue, $\mathrm{C}_{16} \mathrm{H}_{18} \mathrm{ClN}_{3} \mathrm{~S}$ $\left(\varepsilon_{664}=0.61 \times 10^{5} \mathrm{M}^{-1} \mathrm{~cm}^{-1}\right)$;

zinc octakis(cholinyl)phthalocyanine, $\mathrm{ZnPcChol}_{8}$ $\left(\varepsilon_{682}=1.7 \times 10^{5} \mathrm{M}^{-1} \mathrm{~cm}^{-1}\right) ; \quad$ meso-tetrakis[1-(2'bromoethyl)-3-pyridyl]-bacteriochlorin tetra-bromide, (3-PyBrE) ${ }_{4} \mathrm{BCBr}_{4}\left(\varepsilon_{762}=1 \times 10^{5} \mathrm{M}^{-1} \mathrm{~cm}^{-1}\right)$. These PS do not aggregate in a wide range of concentrations and are promising both for photodynamic inactivation of pathogens $[6,8,9,13]$ and for antitumor photodynamic therapy $[10,14,15]$. Fig. 1 shows the normalized spectral contours $A(\lambda)$ of the absorption bands of these PSs (the absorption spectrum at low concentration divided by its maximum value) and the normalized emission spectra $I_{\text {norm }}(\lambda)$ of LEDs (mLED-664, mLED-684, mLED-763, Biospec, Russia), which can be used to excite these PSs (light intensity spectral density divided by the integral intensity).

The estimates of the RAPD in a non-scattering layer with the thickness $L$, obtained in the approximation that the spectral characteristics of PS absorption are independent of its concentration, lead to the following relation characterizing RAPD parameter $\eta$, equal to a ratio between the light energy absorbed in the layer and total light energy illuminating the layer during the PDA process:

$$
\eta=1-\frac{\int_{\lambda_{1}}^{\lambda_{2}} I(\lambda) \times e^{-2.3 D(\lambda)} d \lambda}{\int_{\lambda_{1}}^{\lambda_{2}} I(\lambda) d \lambda}
$$

where

$$
D(\lambda)=\varepsilon A(\lambda) C L
$$

Here $D(\lambda)$ is the optical density of the layer containing PS, $\varepsilon$ and $C$ - extinction and molar concentration of PS, $\lambda_{1}$ and $\lambda_{2}$ are the boundaries of the spectral range in which the spectrum of the radiation source lies, $I(\lambda)$ is the spectral density of the light intensity.

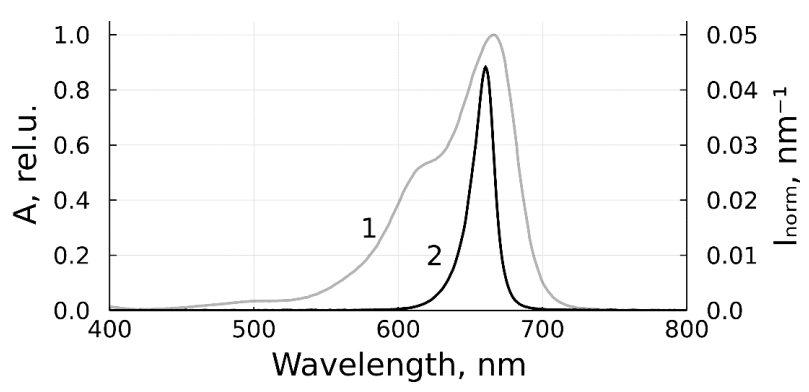

(a)

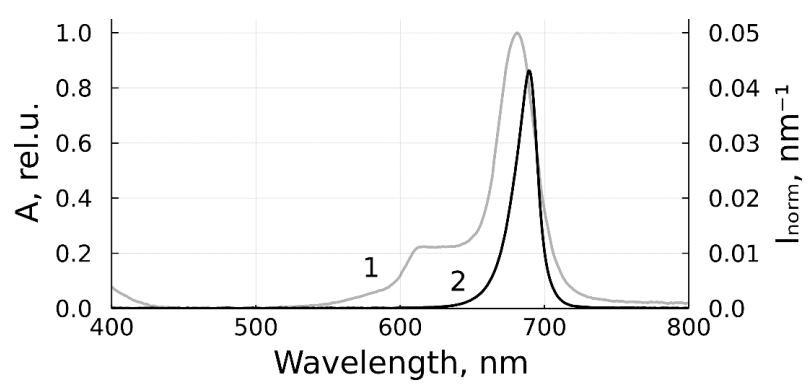

(b)

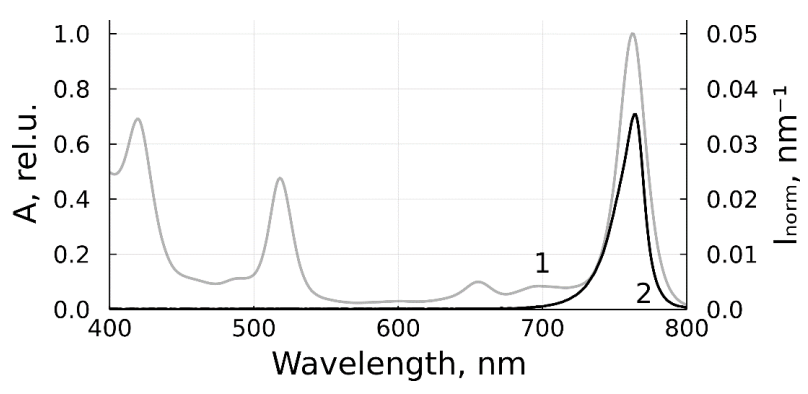

(c)

Fig. 1 Normalized spectral contours (1) of the absorption bands of photosensitizers $\left(\mathrm{a}-\mathrm{C}_{16} \mathrm{H}_{18} \mathrm{ClN}_{3} \mathrm{~S}\right.$, $\left.\mathrm{b}-\mathrm{ZnPcChol}_{8}, \mathrm{c}-(3-\mathrm{PyBrE})_{4} \mathrm{BCBr}_{4}\right)$ and normalized emission spectra (2) of LEDs for their excitation. 
For a broadband light source with a bandpass filter that illuminates in the range from $\lambda_{1}$ to $\lambda_{2}$, the Eq. (1) becomes

$$
\eta=1-\frac{\int_{\lambda_{1}}^{\lambda_{2}} e^{-2.3 D(\lambda)} d \lambda}{\lambda_{2}-\lambda_{1}}
$$

For a monochromatic or narrow-band source, the spectral half-width of which is much smaller than the absorption spectrum half-width, the Eq. (1) is simplified to

$$
\eta=1-e^{-2.3 D(\lambda)}
$$

\section{Results}

The estimates obtained using these formulas show that when thin layers of the studied PS with a concentration below $10 \mu \mathrm{M}$ are irradiated, a significant part of the light energy is not absorbed. Instead, it passes through the sensitized layer. RAPD depends on the PS's extinction, its concentration, the spectral shapes of the PS's absorption and the source emission (Figs. 2,3). The highest values of RAPD are achieved upon monochromatic excitation at a wavelength coinciding with the spectral absorption maximum of the PS. When using LEDs with optimally selected radiation wavelengths, the difference in the value of RAPD is small. However, when using a source whose radiation wavelength is noticeably different from the wavelength of the spectral maximum of the absorption band of the PS (even within the boundaries of the band), RAPD decreases significantly, especially for PS with a narrow absorption band. RAPD of broadband sources with transmission bandpass filters in thin layers is much lower. The values of RAPD for light from such sources can be 1.5-2 times lower than from narrow-band sources.

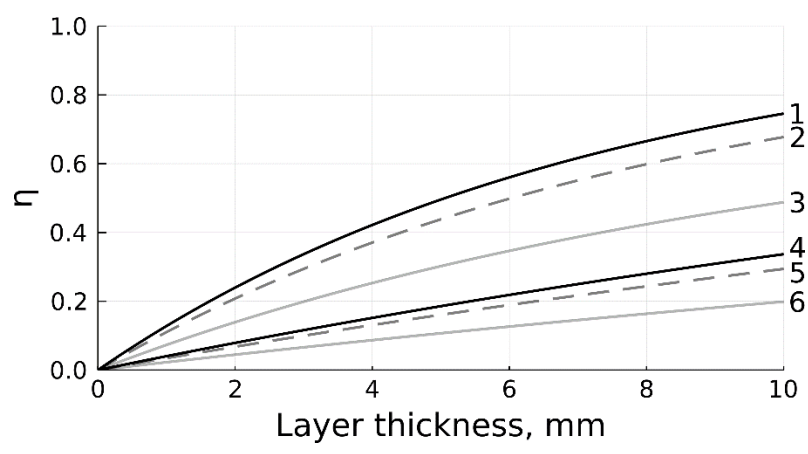

(a)

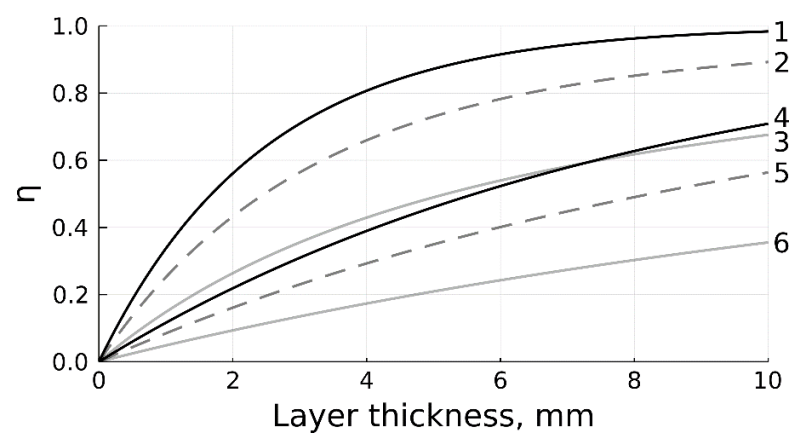

(b)

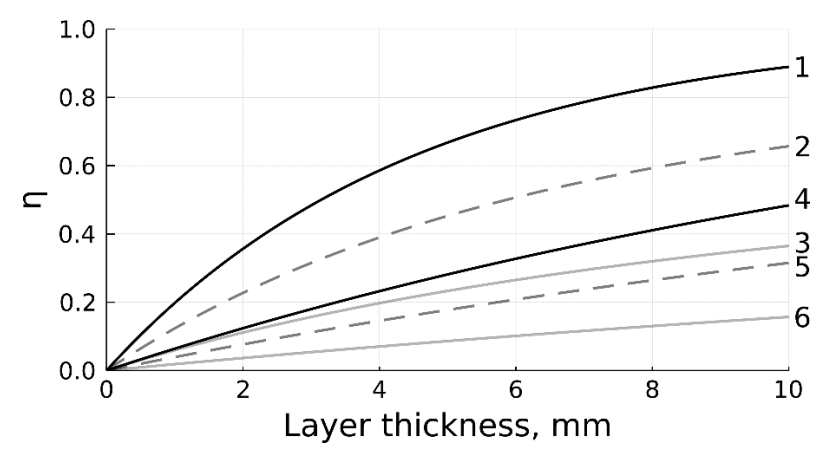

(c)

Fig. 2 Dependence on the layer thickness of RAPD for sources with the wavelength of the maximum radiation coinciding with the wavelength of the spectral maximum of absorption of PS with two concentrations: (a) $\mathrm{C}_{16} \mathrm{H}_{18} \mathrm{ClN}_{3} \mathrm{~S}$, (b) $\mathrm{ZnPcChol} 8$; (c) (3-PyBrE) ${ }_{4} \mathrm{BCBr}_{4} ; 1,2,3$ - concentration of $10 \mu \mathrm{M}, 4,5,6$ - concentration of $3 \mu \mathrm{M}$; 1, 4 - laser; 2, 5 - LED; 3, 6- broadband radiation source with a bandwidth of $100 \mathrm{~nm}$. 


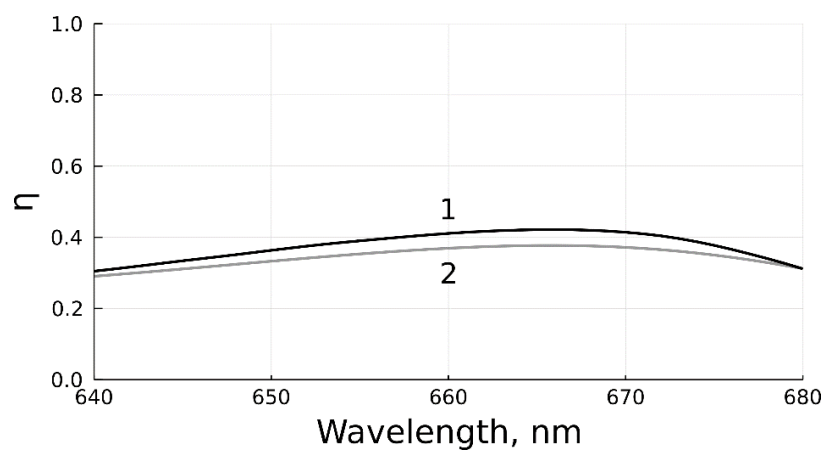

(a)

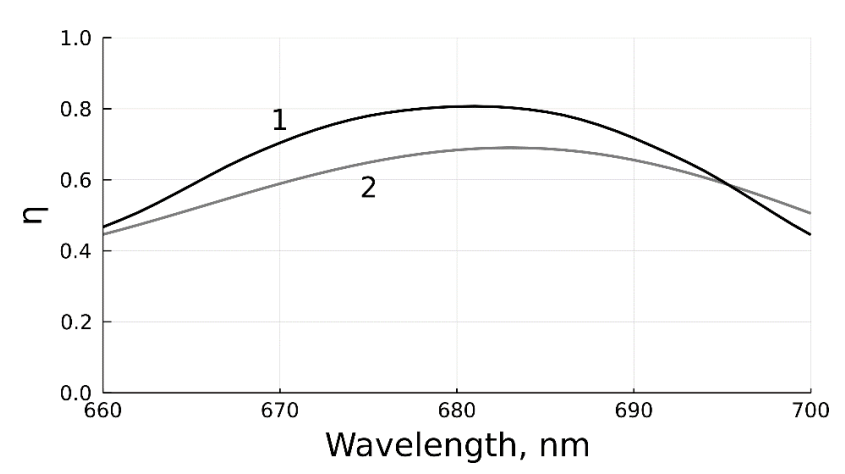

(b)

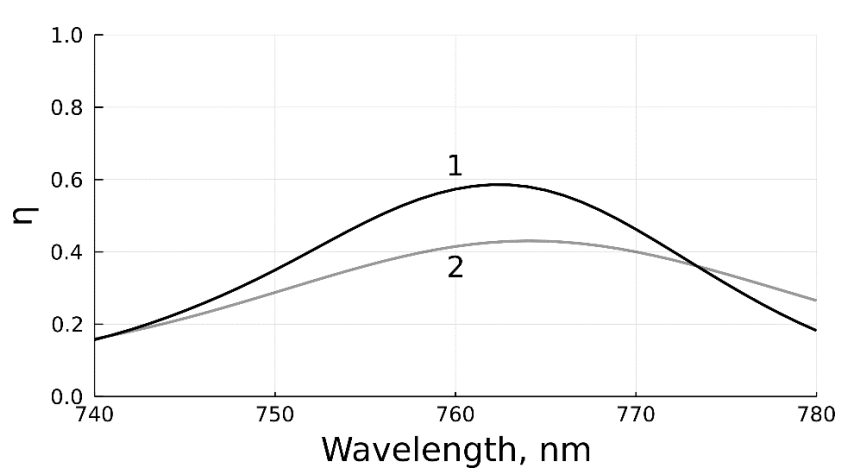

(c)

Fig. 3 RAPD of the sensitized PS layer with a thickness of $5 \mathrm{~mm}$ depending on the wavelength of the spectral maximum of the light source (1 - laser, 2 - LED). PS concentration is $10 \mu \mathrm{M}$ : (a) $\mathrm{C}_{16} \mathrm{H}_{18} \mathrm{ClN}_{3} \mathrm{~S}$, (b) $\mathrm{ZnPcChol}$; (c) (3-PyBrE) ${ }_{4} \mathrm{BCBr} 4$.

\section{Conclusions}

The estimates demonstrate that during PDA in thin sensitized layers, a significant part of the exciting light leaves the layer without being absorbed, and RAPD depends on the layer thickness, PS extinction and concentration, as well as the shape of the exciting light and absorption spectral bands of PS. The loss of a part of the light energy reduces the efficiency of the PDT as a whole. This loss must be taken into account when predicting PDA results and optimizing its tactics, particularly when choosing light sources and parameters of irradiation for photodynamic disinfection of surfaces from viral and bacterial infections $[2,5]$, or clinical photodynamic treatment to local virus-infected foci $[5,6]$. The obtained results are also important for in vitro studies and comparing the photodynamic efficiency of PSs [10, 14] since the values of RAPD are very different for PSs with different extinction, especially when using broadband light sources. However, it should be noted that for in vivo studies of antitumor PDA, it becomes necessary to consider the scattering properties of the medium for evaluating RAPD. Photodynamic effectiveness also becomes more dependent not only on RAPD but on photochemical and photobiological factors as well. High absorption and scattering would also result in greater depth inhomogeneity of PDA in thick sensitized layers or solid tumors, reducing the overall effectiveness of the treatment.

\section{Disclosures}

All authors declare that there is no conflict of interests in this paper.

\section{Acknowledgements}

The research of M. Strakhovskaya, G. Meerovich was funded by RFBR according to the project \#20-04-60084.

The research of E. Akhlyustina, E. Kogan, I. Angelov, E. Borisova was funded by RFBR and BNSF according to the bilateral project (RFBR \#20-52-18008, BNSF \#KP-06-Russia/9-2020). 


\section{References}

1. J. Usuda, T. Inoue, T. Tsuchida, K. Ohtani, S. Maehara, N. Ikeda, Y. Ohsaki, T. Sasaki, and K. Oka, “Clinical trial of photodynamic therapy for peripheral-type lung cancers using a new laser device in a pilot study," Photodiagnosis and Photodynamic Therapy 30, 101698 (2020).

2. H. Abrahamse, M. R. Hamblin, "New photosensitizers for photodynamic therapy," Biochemical Journal 473(4), 347364 (2016).

3. G. A. Meerovich, E. V. Akhlyustina, I. G. Tiganova, E. A. Lukyanets, E. A. Makarova, E. R. Tolordava, O. A. Yuzhakova, I. D. Romanishkin, N. I. Philipova, Yu. S. Zhizhimova, Yu. M. Romanova, V. B. Loschenov, and A. L. Gintsburg, "Novel Polycationic Photosensitizers for Antibacterial Photodynamic Therapy," in Advances in Experimental Medicine and Biology, Springer, New York, 1-19 (2019).

4. M. C. Geralde, I. S. Leite, N. M. Inada, A. C. G. Salina, A. I. Medeiros, W. M. Kuebler, C. Kurachi, and V. S. Bagnato, "Pneumonia treatment by photodynamic therapy with extracorporeal illumination - an experimental model," Physiological Reports 5(5), e13190 (2017).

5. L. D. Dias, V. S. Bagnato, "An update on clinical photodynamic therapy for fighting respiratory tract infections: a promising tool against COVID-19 and its co-infections," Laser Physics Letters 17(8), 083001 (2020).

6. E. Ben-Hur, A. C. E. Moor, H. Margolis-Nunno, P. Gottlieb, M. M. Zuk, S. Lustigman, B. Horowitz, A. Brand, J. Van Steveninck, and T. M. A. R. Dubbelman, "The photodecontamination of cellular blood components: mechanisms and use of photosensitization in transfusion medicine," Transfusion Medicine Reviews 10(1), 15-22 (1996).

7. D. Korneev, O. Kurskaya, K. Sharshov, J. Eastwood, and M. Strakhovskaya, "Ultrastructural Aspects of Photodynamic Inactivation of Highly Pathogenic Avian H5N8 Influenza Virus," Viruses 11(10), 955 (2019).

8. K. Sharshov, M. Solomatina, O. Kurskaya, I. Kovalenko, E. Kholina, V. Fedorov, G. Meerovich, A. Rubin, and M. Strakhovskaya, "The Photosensitizer Octakis(cholinyl)zinc Phthalocyanine with Ability to Bind to a Model Spike Protein Leads to a Loss of SARS-CoV-2 Infectivity In Vitro When Exposed to Far-Red LED," Viruses 13(4), 643 (2021).

9. M. G. Strakhovskaya, G. A. Meerovich, A. N. Kuskov, S. A. Gonchukov, and V. B. Loschenov, "Photoinactivation of coronaviruses: going along the optical spectrum," Laser Physics Letters 17(9), 093001 (2020).

10. R. I. Yakubovskaya, A. D. Plyutinskaya, E. A. Plotnikova, M. A. Grin, and A. F. Mironov, "Comparative in vitro study of different classes of photosensitizers. Pyropheophorbides and chlorines," Russian Journal of Biotherapy 14(1), 43-51 (2015) [in Russian].

11. G. A. Meerovich, E. V. Akhlyustina, I. G. Tiganova, E. A. Lukyanets, E. A. Makarova, E. R. Tolordava, O. A. Yuzhakova, I. D. Romanishkin, N. I. Philipova, Yu. S. Zhizhimova, S. A. Gonchukov, Yu. M. Romanova, and V. B. Loschenov, "Photodynamic inactivation of Pseudomonas aeruginosa bacterial biofilms using new polycationic photosensitizers," Laser Physics Letters 16(11), 115603 (2019).

12. B. W. Pogue, L. Lilge, M. S. Patterson, B. C. Wilson, and T. Hasan, "Absorbed photodynamic dose from pulsed versus continuous wave light examined with tissue-simulating dosimeters,” Applied Optics 36(28), 7257-7269 (1997).

13. V. A. Svyatchenko, S. D. Nikonov, A. P. Mayorov, M. L. Gelfond, and V. B. Loktev, "Antiviral photodynamic therapy: Inactivation and inhibition of SARS-CoV-2 in vitro using methylene blue and Radachlorin," Photodiagnosis and Photodynamic Therapy 33, 102112 (2021).

14. R. I. Yakubovskaya, E. A. Plotnikova, A. D. Plyutinskaya, N. B. Morozova, V. I. Chissov, E. A. Makarova, S. V. Dudkin, E. A. Lukyanets, and G. N. Vorozhtsov, "Photophysical properties and in vitro and in vivo photoinduced antitumor activity of cationic salts of meso-tetrakis(N-alkyl-3-pyridyl)bacteriochlorins," Journal of Photochemistry and Photobiology B: Biology 130, 109-114 (2014).

15. N. B. Morozova, R. I. Yakubovskaya, V. I. Chissov, V. M. Negrimovskiy, and O. A. Yuzhakova, "In vivo photoinduced activity of positively charged zinc phthalocyanine used for photodynamic therapy for malignancies," Russian Journal of Oncology 17(1), 23-28 (2012) [in Russian]. 